\title{
High serum macrophage inflammatory protein-3 $\alpha$ is associated with the early recurrence or metastasis of non-small cell lung cancer following primary pulmonary resection
}

\author{
XIAOPENG ZHANG ${ }^{1,2^{*}}$, AIHONG MENG ${ }^{3 *}$, HUIEN WANG ${ }^{2}$ and XIXIN YAN ${ }^{1,3}$ \\ ${ }^{1}$ Graduate School, Hebei Medical University; ${ }^{2}$ Department of Thoracic Surgery, Hebei Province General Hospital; \\ ${ }^{3}$ Respiratory Division, The Second Hospital of Hebei Medical University, Shijiazhuang, Hebei, P.R. China
}

Received November 24, 2013; Accepted May 23, 2014

DOI: $10.3892 / 01.2014 .2229$

\begin{abstract}
The present study sought to characterize the role of macrophage inflammatory protein-3 $\alpha$ (MIP-3 $\alpha$ ) in non-small cell lung cancer (NSCLC) patients with early recurrence or metastasis after primary pulmonary resection. Follow-up examinations were conducted for 203 NSCLC patients with primary pulmonary resection for two years post-operatively, and data was also collected for 20 healthy subjects. Serum MIP-3 $\alpha$ levels were determined prior to surgery and at post-operative days (PODs) 30, 90 and 180, and the relevant clinical and operative variables were collected. Serum MIP-3 $\alpha$ was measured using a commercially available enzyme-linked immunosorbent assay. There were no significant differences in age, gender and histological type among all groups ( $\mathrm{P}>0.05)$. Serum MIP- $3 \alpha$ levels on POD 180 were significantly higher in the recurrence group than in the non-recurrence group and healthy subjects $(\mathrm{P}=0.001)$. There was no significant difference in the serum MIP-3 $\alpha$ level at PODs 90 and 180 in the patients with or without adjuvant chemotherapy $(\mathrm{P}>0.05)$. The recurrence rate in the high serum MIP-3 $\alpha$ level group was $41.67 \%$, much higher than the $23.53 \%$ observed in the low level group $(\mathrm{P}=0.006)$. The patients with high serum levels of MIP- $3 \alpha$ had a significantly shorter overall recurrence-free time compared with those with low levels $(\mathrm{P}=0.004)$. Multivariate Cox's regression analyses showed that only serum MIP-3 $\alpha$ level was significant, with a hazard ratio of 1.061, a 95\% confidence interval of 1.044-1.078 and a P-value of 0.001. The serum MIP-3 $\alpha$ level in the patients with liver and bone metastases were remarkably higher than those with recurrence at other sites. The high post-operative
\end{abstract}

Correspondence to: Professor Xixin Yan, Respiratory Division, The Second Hospital of Hebei Medical University, 215 Heping Western Road, Shijiazhuang, Hebei 050000, P.R. China E-mail: yanxixin2hospital@hotmail.com

*Contributed equally

Key words: macrophage inflammatory protein-3 $\alpha$, recurrence, non-small cell lung cancer, video-assisted thoracoscopic surgery, metastasis serum MIP-3 $\alpha$ levels were associated with an increased risk of post-operative early recurrence or metastasis in the lung cancer patients, specifically in those with bone or liver metastases.

\section{Introduction}

Lung cancer is the second most frequently occurring cancer in the world, and is the leading cause of cancer-related mortality (1). Non-small cell lung cancer (NSCLC) has a poor prognosis, with a five-year survival rate of $40-50 \%$ in patients with pathological stage I or II disease who undergo a primary resection (2-4). Gender, age, type of surgery, tumor diameter and post-operative $\mathrm{N}$-stage are significant contributing factors to the recurrence risk, with pathological stage and histological type significantly affecting recurrence-free survival $(5,6)$

Increased levels of inflammatory cytokines have been reported as risk factors for recurrence in a number of cancer types (7). Macrophage inflammatory protein-3 (MIP-3 $\alpha$ ), also known as CCL20, has been linked to the propagation of several malignancies, including prostate, hepatic and pancreatic carcinomas, raising the possibility that MIP- $3 \alpha$ plays a role in lung carcinogenesis (8), thereby affecting the prognosis of lung cancer. However, there have been no studies regarding the association between the serum level of MIP-3 $\alpha$ and the prognosis of NSCLC patients following primary resection. The present study sought to characterize the role of MIP-3 $\alpha$ in NSCLC patients with early recurrence or metastasis.

\section{Materials and methods}

Study population. A total of 20 healthy subjects were enrolled, together with 239 NSCLC patients whose diagnosis (pathological Stage I or II, pre-operative assessments) was confirmed by histological examination, between February 2006 and July 2010, at the Hebei Province General Hospital (Shijiazhuang, Hebei, China). From all the patients with lung cancer who underwent pulmonary resections by video-assisted thoracoscopic surgery (VATS), the following patients were excluded: Four patients that were converted to a thoracotomy for bleeding and dense fibrous adhesions; three with pathologically-positive cancer of the bronchial stump; four that presented with surgically unresectable cancer with metastatic lymph nodes; four with pleural disseminations; 
five with chest wall cancer stumps; eight with serious complications or who succumbed; and eight with inadequate follow-up data. The remaining 203 patients with post-operative histopathological stage I or II NSCLC were included in this study. Staging prior to and following the surgery was based on histopathological analysis according to the International Union Against Cancer tumor-node-metastasis staging system (3). The study was approved by the Medical Ethics Committee of Hebei Province General Hospital on human research (code, 2005-123). Informed consent was obtained from each patient. Patients were followed up for two years post-operatively.

Surgical procedures. General anesthesia with double-lumen endotracheal intubation and one-lung ventilation was used in all patients. With the VATS procedure, three working ports were made for the insertion of thoracoscopic instruments, located at the 7 th or 8th intercostal space in the mid-axillary line, the 4th or the 5th intercostal space in the anterior axillary line, and the 8th or the 9th intercostal space in the scapular line. Specimen bags were used for removing the samples. Neither muscles (latissimus dorsi or serratus anterior muscles) nor ribs were cut. During the surgery, the quantity of hemorrhage $(\mathrm{QOH})$ and duration of surgery (DOS) were recorded.

Enzyme-linked immunosorbent assay (ELISA) for serum $M I P-3 \alpha$ level. Blood samples were collected in the morning prior to the surgery and on post-operative days (PODs) 30,90 and 180. Blood samples were also collected from healthy subjects. The serum was obtained by centrifugation at 3,000 x $\mathrm{g}$ for $10 \mathrm{~min}$ and stored at $-80^{\circ} \mathrm{C}$ until analysis. Serum MIP-3 $\alpha$ level was measured using a commercially available ELISA, following the manufacturer's instrctions (RayBiotech, Inc., Norcross, GA, USA). Briefly, 96-well plates coated with the monoclonal mouse anti-human IgG1 MIP3a antibody were incubated with standards at different concentrations, and serum samples at room temperature for $2.5 \mathrm{~h}$. Subsequent to three washes, the wells were incubated with a biotinylated polyclonal goat anti-human MIP3a antibody, at room temperature for $1 \mathrm{~h}$, prepared HRP-conjugated streptavidin for $45 \mathrm{~min}$ at room temperature and TMB One-step Substrate Reagent (RayBiotech, Inc.) at room temperature for $30 \mathrm{~min}$. Enzymatic reactions were developed and the absorbance was measured at $450 \mathrm{~nm}$ in a Multiskan FC microplate (Thermo Fisher Scientifitic, Waltham, MA, USA). Protein levels were calculated according to standard curves.

Statistical analysis. Statistical analysis was performed using SPSS 13 (SPSS, Inc., Chicago, IL, USA). Serum MIP-3 $\alpha$ levels and clinical and operative continuous variables are presented as the mean \pm standard deviation. ANOVA and $\chi^{2}$ tests for independence were used for the comparison of clinical data and serum MIP-3 $\alpha$ levels at each time-point. Independent sample t-tests were employed to investigate the differences in serum MIP-3 $\alpha$ levels between patients with and without adjuvant chemotherapy. The $\chi^{2}$ test was used to examine the recurrence rate between high and low serum MIP-3 $\alpha$ level groups. The Kaplan-Meier method was used to compare recurrence-free survival rates using a log-rank test to examine differences in recurrence free times for high and low serum MIP-3 $\alpha$ level groups. Cox's proportional hazards regression was further employed to estimate the hazard risk ratio of early recurrence

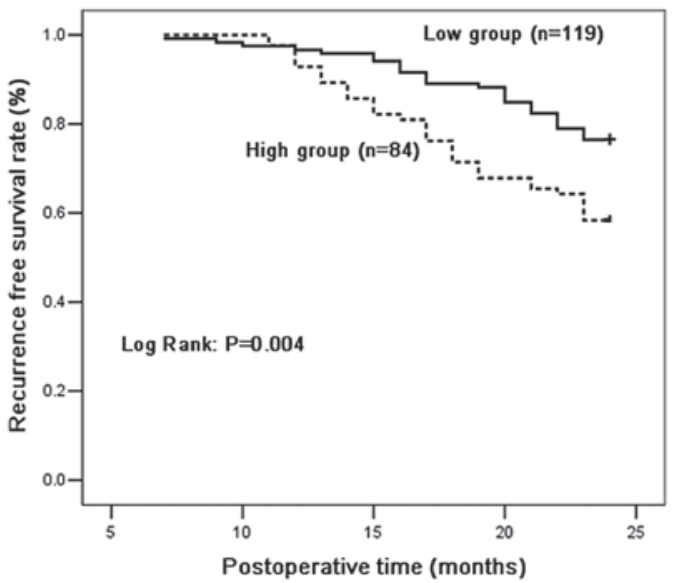

Figure 1. Recurrence-free survival curves for high and low serum MIP-3 $\alpha$ groups. Patients with high serum levels of MIP-3 $\alpha$ had a significantly shorter overall recurrence-free time compared with those with low levels $(\mathrm{P}=0.004)$. MIP-3 $\alpha$, macrophage inflammatory protein- $3 \alpha$.

for levels of serum MIP-3 $\alpha$ and other clinical and operative predictors. ANOVA was used to examine differences in the serum MIP-3 $\alpha$ levels among different metastatic or recurrent position groups. $\mathrm{P}<0.05$ was considered to indicate a statistically significant difference.

\section{Results}

Association between tumor recurrence or metastasis and clinicopathological parameters. A total of 203 patients were reviewed for up to two years post-operatively. In total, 63 patients experienced recurrence of NSCLC, with a two-year recurrence rate of $31.0 \%$. The serum MIP-3 $\alpha$ levels on POD 180 were significantly higher in the recurrence group than those in the non-recurrence group and healthy subjects $(\mathrm{P}=0.001)$. The adjuvant chemotherapy rate was low in the non-recurrence group $(\mathrm{P}=0.035)$, while there were no significant differences in age, gender, QOH, DOS and histological type between the recurrence and non-recurrence groups $(\mathrm{P}>0.05)$ (Table I) .

\section{Adjuvant chemotherapy has no significant effect on serum} $M I P-3 \alpha$ level. Adjuvant chemotherapy was carried out in certain patients after POD 30. To evaluate whether adjuvant chemotherapy contributes to the value of serum MIP- $3 \alpha$, the serum MIP-3 $\alpha$ levels were compared at PODs 90 and 180 in the recurrence and non-recurrence groups, respectively, considering adjuvant chemotherapy as a main factor for the recurrence and non-recurrence groups. It was found that there was no significant difference in the serum MIP-3 $\alpha$ level at PODs 90 and 180 in all groups $(\mathrm{P}>0.05)$ (Tables II and III).

High serum MIP-3a level contributes to a high recurrence risk. The patients were divided into high and low serum MIP-3 $\alpha$ level groups by the median value of the serum MIP-3 $\alpha$ level $(57 \mathrm{pg} / \mathrm{ml})$ at POD 180. The recurrence rate in the high serum MIP-3 $\alpha$ level group was significantly higher than that in the low serum MIP-3 $\alpha$ level group $(\mathrm{P}=0.006)$ (Table IV).

Recurrence-free survival analysis. Recurrence-free survival curves for the high and low MIP-3 $\alpha$ groups are shown 
Table I. Clinical data and serum MIP-3 $\alpha$ level in healthy subjects, recurrence and non-recurrence groups.

\begin{tabular}{lccr}
\hline Patient characteristics & Healthy & Rec (-) & Rec (+) \\
\hline Total, n & 20 & 140 & 63 \\
Age, years & $62.75 \pm 9.76$ & $62.37 \pm 8.94$ & $62.05 \pm 9.88$ \\
Gender (male/female), n & $10 / 10$ & $81 / 59$ & $39 / 24$ \\
Histological type (Ad/Sq/LC/other), n & & $75 / 61 / 2 / 2$ & $33 / 27 / 2 / 1$ \\
Adjuvant/non-adjuvant chemotherapy, n & & $45 / 95$ & $30 / 33$ \\
QOH, ml & & $99.66 \pm 28.59$ & $94.46 \pm 26.24$ \\
DOS, min & & $165.89 \pm 35.19$ & $162.77 \pm 32.16$ \\
MIP-3 $\alpha$, pg/ml & $51.06 \pm 8.19$ & & 0.930 \\
Pre-operatively & & $48.10 \pm 12.35$ & 0.035 \\
POD 30 & & $49.79 \pm 10.54$ & $51.25 \pm 104$ \\
POD 90 & & $52.83 \pm 13.66$ & $47.66 \pm 11.63$ \\
POD 180 & & $53.96 \pm 10.38$ & $49.74 \pm 11.55$ \\
\hline
\end{tabular}

MIP-3 $\alpha$, macrophage inflammatory protein-3 $\alpha$; Rec (+), recurrence group; Rec (-), non-recurrence group; Ad, adenocarcinoma; Sq, squamous cell carcinoma; LC, large cell carcinoma; QOH, quantity of hemorrhage; DOS, duration of surgery; POD, post-operative day.

Table II. Serum MIP-3 $\alpha$ level (pg/ml) in the recurrence group.

\begin{tabular}{lccc}
\hline Day & Adjuvant chemotherapy $(\mathrm{n}=30)$ & Non-adjuvant chemotherapy $(\mathrm{n}=33)$ & 0.359 \\
\hline POD 90 & $50.75 \pm 10.13$ & $48.51 \pm 9.15$ & 0.192 \\
POD 180 & $68.94 \pm 12.11$ & $72.49 \pm 10.23$ & 0.23 \\
\hline
\end{tabular}

MIP-3 $\alpha$, macrophage inflammatory protein-3 $\alpha$; POD, post-operative day.

Table III. Serum MIP-3 $\alpha$ level (pg/ml) in the non-recurrence group.

\begin{tabular}{lccc}
\hline Day & Adjuvant chemotherapy $(\mathrm{n}=45)$ & Non-adjuvant chemotherapy $(\mathrm{n}=95)$ & $\mathrm{P}-\mathrm{value}$ \\
\hline POD 90 & $53.31 \pm 11.12$ & $49.59 \pm 11.75$ & 0.077 \\
POD 180 & $55.83 \pm 9.81$ & $52.59 \pm 10.75$ & 0.089
\end{tabular}

MIP-3 $\alpha$, macrophage inflammatory protein-3 $\alpha$; POD, post-operative day.

Table IV. Recurrence rate in the high and low serum MIP-3 $\alpha$ level groups.

\begin{tabular}{|c|c|c|c|c|}
\hline serum MIP-3 $\alpha$ level at POD $180, \mathrm{pg} / \mathrm{ml}$ & Number & $\operatorname{Rec}(-)$ & $\operatorname{Rec}(+)$ & Rate, $\%$ \\
\hline Low $(<57), \mathrm{n}$ & 119 & 91 & 28 & 23.53 \\
\hline $\operatorname{High}(>57), \mathrm{n}$ & 84 & 49 & 35 & 41.67 \\
\hline
\end{tabular}

$\mathrm{P}=0.006$. MIP-3 $\alpha$, macrophage inflammatory protein-3 $\alpha$; Rec (+), recurrence group; Rec (-), non-recurrence group; POD, post-operative day.

in Fig. 1. Patients in the high and low groups had two year recurrence-free rates of 76.5 and $58.3 \%$, respectively. There were significant differences in overall recurrence-free survival between patients with high and low serum MIP-3 $\alpha$ level. Patients with high serum levels of MIP- $3 \alpha$ had a significantly shorter overall recurrence-free time compared with those with low levels $(\mathrm{P}=0.004)$.

Multivariate Cox's regression analysis. The study further investigated the independent effects of MIP- $3 \alpha$ on early 
Table V. Recurrence factors in Cox's proportional hazards model.

\begin{tabular}{llr}
\hline Patient characteristics & HR $(95 \% \mathrm{CI})$ & P-value \\
\hline Age, years & $0.989(0.962-1.017)$ & 0.443 \\
Gender (male/female) & $0.990(0.544-1.803)$ & 0.974 \\
Histological type (Ad/Sq/LC/Other) & $0.508(0.989-2.298)$ & 0.076 \\
Adjuvant/non-adjuvant chemotherapy & $1.405(0.976-1.985)$ & 0.069 \\
QOH, ml & $0.996(0.987-1.135)$ & 0.407 \\
DOS, min & $1.002(0.994-1.010)$ & 0.624 \\
MIP-3 $\alpha$, pg/ml & & \\
Pre-operatively & $1.003(0.985-1.021)$ & 0.730 \\
POD 30 & $1.012(0.986-1.038)$ & 0.366 \\
POD 90 & $1.002(0.984-1.020)$ & 0.821 \\
POD 180 & $1.061(1.044-1.078)$ & 0.001 \\
\hline
\end{tabular}

HR, hazard ratio; CI, confidence interval; Ad, adenocarcinoma; Sq, squamous cell carcinoma; LC, large cell carcinoma; QOH, quantity of hemorrhage; DOS, duration of surgery; MIP-3 $\alpha$, macrophage inflammatory protein-3 $\alpha$; POD, post-operative day.

Table VI. Post-operative tumor recurrence or metastasis site and serum MIP-3 $\alpha$ level.

\begin{tabular}{lcc}
\hline Recurrence site & Number $(\mathrm{n}=63)$ & Serum MIP-3 $\alpha$ level at POD 180, pg/ml \\
\hline Lung & 10 & $65.92 \pm 9.15$ \\
Lymph node & 11 & $64.45 \pm 8.49$ \\
Brain & 7 & $67.65 \pm 8.24$ \\
Bone & 7 & $85.10 \pm 9.95^{\mathrm{a}}$ \\
Liver & 6 & $87.31 \pm 11.12^{\mathrm{a}}$ \\
Pleural dissemination & 2 & $68.85 \pm 2.33$ \\
Lung+mediastinal lymph node & 7 & $67.95 \pm 8.40$ \\
Lung+brain & 7 & $66.67 \pm 9.74$ \\
Lung+bone & 6 & $88.34 \pm 12.46^{\mathrm{a}}$
\end{tabular}

${ }^{a} \mathrm{P}<0.05$ vs. other recurrence sites. The patients with pleural dissemination were excluded in analysis due to the small sample size. MIP- $3 \alpha$, macrophage inflammatory protein- $3 \alpha$; POD, post-operative day.

recurrence or metastasis with respect to age, gender, $\mathrm{QOH}$, DOS, histological type and adjuvant chemotherapy rate using Cox's regression models. Only the serum MIP-3 $\alpha$ level on POD 180 was a significant predictor for early recurrence or metastasis. The MIP-3 $\alpha$ level on POD 180 had a hazard ratio of 1.061, with a 95\% confidence interval of 1.044-1.078 and a $\mathrm{P}$-value of 0.001. In the multivariate Cox's regression analyses, only the serum MIP-3 $\alpha$ level was significant. Other factors were not independent predisposing factors for post-operative early recurrence or metastasis (Table V).

Serum MIP-3a level of varying recurrence or metastasis sites. In the two years of follow-up, the earliest and average recurrence times were 7 and 17.25 months after surgery, respectively. The serum MIP-3 $\alpha$ level was also investigated at POD 180 in the patients with varying recurrence or metastasis sites, as shown in Table VI. The serum MIP-3 $\alpha$ levels in the patients with liver and bone metastases were significantly higher than those in the patients with other sites of recurrence $(\mathrm{P}<0.05)$. The patients with pleural dissemination had to be excluded from the analysis due to the small sample size.

\section{Discussion}

MIP-3 $\alpha$ is the only cytokine known to interact with CC chemokine receptor 6 (CCR6), a property shared with the antimicrobial $\beta$-defensins. The ligand-receptor pair MIP-3 $\alpha$-CCR6 is responsible for the chemoattraction of immature dendritic cells, effector/memory T cells and B cells, and plays a critical role in cancer and rheumatoid arthritis (9). MIP-3 $\alpha$ has been linked to the development of malignant tumors. The high expression of MIP-3 $\alpha$ has been reported in cancers of the colon (10), pancreas (11), prostate (12), nasopharynx (13) and liver (14). There is evidence that MIP-3 $\alpha$ enhances tumor growth in numerous types of cancer $(15,16)$. Moreover, MIP- $3 \alpha$ is considered to be involved in carcinogenesis, angiogenesis and invasion $(17,18)$. 
Surgical procedures are an important primary approach for the treatment of early-stage lung cancer patients. However, metastasis and recurrence are the most common risks for treatment failure following surgery, with a reported $50 \%$ recurrence rate of lung cancer within one year in the treatment of NSCLC (19).

In the present study, it was found that there was a higher post-operative level of MIP-3 $\alpha$ (POD 180) and a relatively higher adjuvant chemotherapy rate NSCLC patients (post-operative histopathological stage I or II) with recurrence or metastasis. Consistent with this finding, Kirshberg et al (20) reported that the MIP-3 $\alpha / C C R 6$ axis promoted NSCLC disease progression. However, in the multivariate Cox's regression analyses of the present study, only serum MIP-3 $\alpha$ level (POD 180) was significant for post-operative early recurrence or metastasis, specifically for bone or liver metastasis. The study indicated that a high post-operative level of MIP-3 $\alpha$ (POD 180) was significantly associated with the early recurrence or metastasis of NSCLC. This is the first study to identify the serum MIP-3 $\alpha$ immune response marker as a predictor for the early post-operative recurrence of NSCLC. The study highlighted the fact that the post-operative MIP-3 $\alpha$ level may therefore be a useful marker to determine the requirement for adjunctive anti-cancer therapy, and the fact that the MIP-3 $\alpha /$ CCR6/IL-17 axis should be investigated further as a potential novel therapeutic target.

To the best of our knowledge, no previous study has examined the post-operative value of serum MIP-3 $\alpha$ in NSCLC patients with adjuvant chemotherapy. In the present study, adjuvant chemotherapy was applied in certain patients after POD 30. It was found that there was no significant difference in the serum MIP-3 $\alpha$ level at PODs 90 and 180 in all patients. This indicated that MIP-3 $\alpha$ was independent of adjuvant chemotherapy, which was consistent with the result of the multivariate Cox's regression analyses. Iwata et al (21) also showed that the serum MIP-3 $\alpha$ status was an independent prognostic factor for overall CRC patients regardless of therapeutic interventions.

In statistical analyses, the patients of the present study were divided into low and high groups according the serum MIP-3 $\alpha$ level following primary pulmonary resection. The patients with a high serum MIP-3 $\alpha$ level had a higher recurrence rate than the patients with a low level. Recurrence-free survival curves showed that there were significant differences in recurrence-free survival between the two groups. This indicated that post-operative patients with high serum MIP-3a levels have a high risk of tumor recurrence or metastasis.

In further experiments, the serum MIP-3 $\alpha$ levels were studied in patients with recurrence and different recurrence or metastasis sites. The results showed the the serum MIP-3a levels were significantly higher in the patients with liver and bone metastases. Iwata et al (21) also reported that the serum MIP-3 $\alpha$ status was significantly associated with synchronous liver metastasis and was as independent predictive factor for liver metastasis.

However, several limitations, including the relatively small sample and the requirement for validation studies in independent samples, exist in this study. More studies are required to further clarify the association between MIP-3 $\alpha$ and recurrence or metastasis in NSCLC patients.

In conclusion, in the present study, high post-operative serum MIP-3 $\alpha$ levels were associated with an increased risk of post-operative early recurrence or metastasis in NSCLC patients (post-operative histopathological stage I or II), specifically in those with bone or liver metastases.

\section{Acknowledgements}

The authors would like to thank their colleagues in the Department of Thoracic Surgery (Hebei Province General Hospital) for collecting the samples and Hanying Xing and Zhijuan Hu for providing technical assistance.

\section{References}

1. Parkin DM, Bray F, Ferlay J and Pisani P: Global cancer statistics, 2002. CA Cancer J Clin 55: 74-108, 2005.

2. Goya T, Asamura H, Yoshimura H, et al; Japanese Joint Committee of Lung Cancer Registry: Prognosis of 6644 resected non-small cell lung cancers in Japan: A Japanese lung cancer registry study. Lung Cancer 50: 227-234, 2005.

3. Mountain CF: Revisions in the International System for Staging Lung Cancer. Chest 111: 1710-1717, 1997.

4. Scagliotti GV and Novello S: Adjuvant therapy in completely resected non-small-cell lung cancer. Curr Oncol Rep 5: 318-325, 2003.

5. Choi YS, Shim YM, Kim K and Kim J: Pattern of recurrence after curative resection of local (stage I and II) non-small cell lung cancer: difference according to the histologic type. J Korean Med Sci 19: 674-676: 2004.

6. Hjelde H, Sundstrøm S, Ødegård A, et al: Recurrence and survival after surgical treatment of lung cancer. Tidsskr Nor Laegeforen 130: 25-28, 2010 (In Norwegian).

7. Jiang J, Goel R, Schmechel S, et al: Pre-conditioning cryosurgery: Cellular and molecular mechanisms and dynamics of tnf- $\alpha$ enhanced cryotherapy in an in vivo prostate cancer model system. Cryobiology 61: 280-288, 2010.

8. Ghadjar P, Rubie C, Aebersold DM and Keilholz U: The chemokine CCL20 and its receptor CCR6 in human malignancy with focus on colorectal cancer. Int J Cancer 125: 741-745, 2009.

9. Schutyser E, Struyf S and Van Damme J: The CC chemokine CCL20 and its receptor CCR6. Cytokine Growth Factor Rev 14: 409-426, 2003.

10. Brand S, Olszak T, Beigel F, et al: Cell differentiation dependent expressed CCR6 mediates ERK-1/2, SAPK/JNK, and Akt signaling resulting in proliferation and migration of colorectal cancer cells. J Cell Biochem 97: 709-723, 2006.

11. Rubie C, Frick VO, Ghadjar P, et al: CCL20/CCR6 expression profile in pancreatic cancer. J Transl Med 8: 45, 2010.

12. Ghadjar P, Loddenkemper C, Coupland SE, et al: Chemokine receptor CCR6 expression level and aggressiveness of prostate cancer. J Cancer Res Clin Oncol 134: 1181-1189, 2008.

13. Chang KP, Hao SP, Chang JH, et al: Macrophage inflammatory protein-3alpha is a novel serum marker for nasopharyngeal carcinoma detection and prediction of treatment outcomes. Clin Cancer Res 14: 6979-6987, 2008.

14. Rubie C, Frick VO, Wagner M, et al: Enhanced expression and clinical significance of CC-chemokine MIP-3 alpha in hepatocellular carcinoma. Scand J Immunol 63: 468-477, 2006.

15. Fujii H, Itoh Y, Yamaguchi K, et al: Chemokine CCL20 enhances the growth of HuH7 cells via phosphorylation of p44/42 MAPK in vitro. Biochem Biophys Res Commun 322: 1052-1058, 2004.

16. Liu J, Zhang N, Li Q, et al: Tumor-associated macrophages recruit $\mathrm{CCR}^{+}$regulatory $\mathrm{T}$ cells and promote the development of colorectal cancer via enhancing CCL20 production in mice. PLoS One 6: e19495, 2011.

17. Slettenaar VI and Wilson JL: The chemokine network: a target in cancer biology? Adv Drug Deliv Rev 58: 962-974, 2006.

18. Tsai ST, Chien IH, Shen WH, et al: ENO1, a potential prognostic head and neck cancer marker, promotes transformation partly via chemokine CCL20 induction. Eur J Cancer 46: 1712-1723, 2010.

19. Lee HJ, Jo J, Son DS, et al: Predicting recurrence using the clinical factors of patients with non-small cell lung cancer after curative resection. J Korean Med Sci 24: 824-830, 2009.

20. Kirshberg S, Izhar U, Amir G, et al: Involvement of CCR6/CCL20/IL-17 axis in NSCLC disease progression. PLoS One 6: e24856, 2011.

21. Iwata T, Tanaka K, Inoue Y, et al: Macrophage inflammatory protein-3 alpha (MIP-3 $\alpha$ ) is a novel serum prognostic marker in patients with colorectal cancer. J Surg Oncol 107: 160-166, 2013. 